\title{
INTERCÂMBIO EDUCACIONAL INTERNACIONAL NA MODALIDADE DOUTORADO SANDUÍCHE EM ENFERMAGEM: RELATO DE EXPERIÊNCIA
}

\author{
International Educational Exchange in Nursing \\ Sandwich Doctorate Modality: Experience Report \\ Intercambio Educacional Internacional en la Modalidad \\ Doctorado Sándwich: Relato de Experiencia
}

Kleyde Ventura de Souza ${ }^{1}$

\begin{abstract}
Resumo
Relato de experiência de estágio de Doutorado Sanduíche oferecido pela Coordenação de Aperfeiçoamento de Pessoal de Nível Superior, realizado na Faculté des Sciences Infirmières/Université de Montreal, no período de agosto a dezembro de 2004. 0 estágio teve como enfoque 0 aprofundamento dos aspectos conceituais e práticos relacionados à saúde da mulher e seus direitos sexuais e reprodutivos. Este artigo tem como propósito apresentar as atividades realizadas e despertar o interesse dos estudantes de doutorado dos programas brasileiros de pós-graduação nessa modalidade de intercâmbio. Destacam-se a participação em cursos, reuniões de grupos de pesquisas e em eventos científicos; visitas técnicas e troca de experiências com pesquisadores e integrantes de organizações sociais e conferências. Com esta experiência, verificou-se a importância da cooperação entre pesquisadores e instituições do Brasil e do exterior e fortaleceu-se o compromisso com um projeto de emancipação profissional e social, por meio da pesquisa e deste tipo de cooperação.
\end{abstract}

Palavras-chave: Intercâmbio Educacional Internacional. Instituições de Ensino Superior. Grupos de Pesquisa. Saúde da Mulher.

Abstract

A report of a training experience of a Sandwich Doctorate offered by the Students' Improvement Coordenation of Higher Education made at Faculté des Sciences Infirmières/Université de Montreal, from August to December, 2004. The training focused the deepening of conceptual and practical concepts related to the women's health and their sexual and reproductive rights. This article aims to present the activities made to raise the interest of doctorate students in the post-graduation Brazilian programs in this kind of exchange. The attendance in courses, research groups and scientific events meetings, technical visits and experience exchange with researchers and members of social organizations, and conferences are emphasized. The importance of cooperation between researchers and institutions in Brazil as well as in other countries was verified with this experience and it consolidated the commitment with a professional and social emancipation project by means of research and by this kind of cooperation.

Keywords: International Educational Exchange. Higher education institutions. Research groups. Women's Health.

\section{Resumen}

El artículo relaciona a la experiencia del entrenamiento del Doctorado Sándwich, ofrecido por Cordenación de Perfeccionamiento de Personal de Nivel Superior y realizado en la Faculté de Sciences Infirmières/Université de Montreal, de agosto al diciembre de 2004. El entrenamiento enfocó en profundizar los aspectos conceptuales y prácticos relacionados con la salud de la mujer y con sus derechos sexuales y reproductivos. Este artículo se trata de presentar las actividades realizadas e inspirar el interés de estudiantes de los programas brasileños de la post-grado en esta modalidad del intercambio. Este intercambio era distinguido por la participación en cursos, en reuniones de grupos de investigación y en conferencias científicos, y en las visitas técnicas y el intercambio de experiencias entre investigadores y miembros de organizaciones social. La experiencia confirmó la importancia de cooperación entre investigadores e instituciones de Brasil y del exterior, mientras que el compromiso de cooperación se fortifica con un proyecto de la emancipación profesional y social.

Palabras clave: Intercambio educacional internacional. Instituciones de enseñanza superior. Grupos de investigación. Salud de la mujer. 


\section{CONSIDERAÇÕES INICIAIS}

A Coordenação de Aperfeiçoamento de Pessoal de Nível Superior do Ministério da Educação (CAPES/MEC) no cumprimento do seu papel de aperfeiçoamento da formação acadêmica em nosso país, por meio de um de seus programas de concessão de bolsas de estudo, no exterior, oferece a oportunidade de realização de estágio a estudantes regularmente matriculados, em cursos de doutorado no Brasil. Esta modalidade de intercâmbio, denominada Estágio de Doutorado, possibilita ao doutorando desenvolver parte de sua pesquisa em instituição estrangeira de reconhecida excelência, a um só tempo. Este procedimento visa fomentar a cooperação entre pesquisadores e instituições do país e do exterior. A Bolsa de Estágio de Doutorando da CAPES/MEC é conhecida como Doutorado Sanduíche e destina-se a doutorandos de comprovado bom desempenho acadêmico. Complementar à formação de docentes e pesquisadores de alto nível, em consonância com os programas de pós-graduação no Brasil, consolida a colaboração entre grupos brasileiros e estrangeiros na realização de pesquisa e formação de novos doutores. Nessa modalidade de estágio, o estudante tem a possibilidade de desenvolver suas atividades no exterior, por um período de 4 até 12 meses. ${ }^{1}$

É com base nos propósitos descritos e no interesse de que tal possibilidade seja aproveitada, amplamente, pelos doutorandos de Enfermagem, que a experiência da realização de estágio de Doutorado Sanduíche caracteriza-se na construção do presente artigo e tem como objetivo apresentar as atividades realizadas e chamar a atenção para a importância destas atividades para a concretização da pesquisa que originou o interesse em realizar este estágio. Pretende-se ainda despertar o interesse dos estudantes de doutorado dos programas de pós-graduação do Brasil, para realização deste tipo de estágio, em particular, das(os) estudantes do Programa de Pós-Graduação da Escola de Enfermagem Anna Nery, da Universidade Federal do Rio de Janeiro EEAN/UFRJ, instituição a qual estivemos vinculadas entre os anos de 2002-2005, para realização de Curso de Doutorado, sob orientação da Profa. Dra . Maria Antonieta Rubio Tyrrella.

0 estágio em tela foi realizado na Faculté des Sciences Infirmières/Université de Montréal-UdeM, durante o período de agosto a dezembro de 2004, sob a orientação do Profo Michel Perreualt'1. Para realizá-lo, o candidato à bolsa de estudos do Programa de Doutorado-Sanduíche no exterior, oferecido pela CAPES/MEC, envia a este órgão, sob a forma de projeto, um plano de atividade, que é submetido à análise de pares, como requisito parcial de aprovação para entrada no Programa. Neste caso, o Plano de Atividades incluiu: troca de experiências com pesquisadores e profissionais de serviços de saúde; participação em discussões multidisciplinares; seminários; visitas técnicas; observações de campo, além da realização de cursos.

Nesta (inter)ação seguimos para Montreal/Canadá, beneficiando-nos do Acordo Acadêmico existente entre a EEAN/UFRJ e a Faculté des Sciences Infirmières/UdeM, o qual engloba um programa de intercâmbio de alunos. Assim, de forma inédita, tivemos a oportunidade de usufruir do acordo de cooperação do Programa de Pós-graduação da EEAN e experimentar os benefícios da modalidade de estudo oferecida e financiada pela CAPES/MEC.

\section{A Interface entre o Currículo Formal e o “Currículo Oculto" da Escola de Enfermagem Anna Nery}

A EEAN/UFRJ, criada sobre os princípios do Sistema Nightingale, completou em, 2003, 80 anos. 0 Programa de Pós-Graduação e Pesquisa da EEAN/UFRJ possui oito linhas de pesquisa, entre elas a Saúde da Mulher. Esta linha encontra-se inserida no Núcleo de Pesquisa em Enfermagem do Departamento Materno Infantil (NUPESM/DEMI), no qual participamos na condição de discente, culminando com a defesa da tese intitulada "A Saúde da Mulher e seus Direitos Sexuais e Reprodutivos em um Círculo de Cidadania".

Nesta oportunidade, foi possível vivenciar no campo do fazer-saber-fazer pesquisa, o "Padrão Ana Néri" ${ }^{3}$ que, a nosso ver conjugou a arte e a ciência de pesquisar em Enfermagem. Assim, conjugamos o aprendizado (objetivo) do currículo formal da EEAN, o qual traduz o próprio "modo de ser" dessa instituição, às apreensões (subjetivas) do currículo "oculto", que nos encaminhou no sentido do diálogo intenso com a realidade e do fortalecimento da idéia/ação de pesquisar como um processo emancipatório e comprometido com as transformações sociais.

Este relato de experiência trata-se, então, da síntese do relatório enviado e aprovado pela agência financiadora, o qual foi realizado com base em documentação reunida ao longo do estágio. Para organização dos dados, os documentos foram classificados e ordenados de acordo com o seu gênero: documentação audiovisual; fonográfica; iconográfica e textualc.

\section{ATIVIDADES REALIZADAS: A DINÂMICA DE DESCOBERTAS DOS ESPAÇOS, DAS PESSOAS E DO OBJ ETO DE PESQUISA}

As atividades realizadas voltaram-se, primordialmente, para o acompanhamento de serviços e grupos de pesquisa do Quebec, com orientação multidisciplinar e ações voltadas à compreensão da influência da especificidade do gênero feminino na promoção e proteção da saúde das mulheres, considerando o contexto das transformações sociais, culturais, econômicas e do sistema de saúde.

Mais especificamente, buscamos dar conta dos objetivos propostos no plano de trabalho, que incluíam: a) verificar como os grupos de pós-graduação no Quebec (Canadá) tratam as questões de cidadania e direitos reprodutivos das mulheres nos serviços de saúde; b) realizar aprofundamento teórico de estudos da enfermagem na área da saúde da mulher, com ênfase nos aspectos relacionados à cidadania feminina; c) avançar nos aspectos conceituais relacionados aos direitos reprodutivos e de cidadania das mulheres. 0 alcance desses objetivos englobou um conjunto de ações: realização de cursos; participação em reuniões de grupos de pesquisas e em eventos científicos e visitas técnicas. Para melhor compreensão das atividades enunciadas, descrevemos cada uma delas a seguir. 


\section{Atividades realizadas sob a forma de Cursos}

0 curso S0I 6127 - L'Approche communautaire tratou-se do estudo sistemático da natureza e dos fundamentos do cuidado de enfermagem na comunidade. Apresentou conceitos epidemiológicos, teóricos e modelos psicossociais e educativos utilizados no campo da saúde da comunidade, junto às populações-alvo nos diversos ciclos da vida. Sua realização favoreceu a ampliação de nosso conhecimento em termos de modelos/abordagens no campo da saúde da comunidade. As discussões, leituras e reflexões oportunizaram o fortalecimento de minha compreensão de saúde como um evento social, coletivo, determinado historicamente pelas condições e modos de vida dos distintos grupos da população, para além do patrimônio biológico e genético. Nesse sentido, compreendemos de fundamental importância a superação de concepções do modo tradicional de fazer-pensar-construir saúde, e a pesquisa aparece como um importante instrumento para contribuir com esse movimento de reorientação tanto no campo do conhecimento, como da prática.

Vale ressaltar que a CAPES/MEC não se responsabiliza com custos referentes a créditos, cursos e disciplinas para alunos de doutorado sanduíche. Ainda assim, fomos admitidas como alunas regulares da UdeM. Nessa condição, nos foi permitido acesso a todos os serviços oferecidos a estudantes regulares, e obtivemos a isenção de taxas de escolaridade, normalmente exigidas de alunos(as) que não se encontram nessa situação. Tal fato ocorreu devido à relação de parceria expressa por meio do acordo acadêmico já citado e do empenho pessoal do co-orientador estrangeiro junto a Direction des Relations Internationales da UdeM, no sentido de fazer valer o intercâmbio mencionado, uma vez que a concretização do mesmo, até aquela data, era inédita.

\section{Atividades realizadas sob a forma de Seminários}

0 GRASP, formado em 1984, constitui-se num grupo de pesquisa multidisciplinar, vinculado à UdeM e reúne um conjunto de pesquisadores, profissionais de pesquisa e estudantes de pós-graduação interessados na análise das relações sociais no âmbito da saúde e do bem-estar. No GRASP vivenciamos o contexto de um "laboratório de pesquisa", na medida em que convivemos com professores e estudantes de diversas disciplinas, entre elas: sociologia; psicologia; história, economia; epidemiologia; ética em pesquisa e na saúde. Esta convivência possibilitou a troca de informações e experiências, quanto à realização de pesquisas e ampliação de nosso conhecimento acerca de referenciais teóricos e metodológicos, no âmbito da promoção da saúde.

\section{Atividades realizadas sob a forma de participação em Eventos Científicos}

A Deuxième Conferénce Cannadienne sur l'Alphabetisation et la Santé foi promovida pela Association Canadienne de Santé Publique (ACSP), que é uma associação nacional, independente e sem fins lucrativos. Representa a saúde pública no Canadá e mantém ligações estreitas com a comunidade de saúde pública, em âmbito internacional. Neste evento tivemos a oportunidade de participar de uma oficina que nos possibilitou conhecer 0 conceito de alfabetização e saúde, bem como, reconhecer as diferenças e similaridades entre este campo de prática e de conhecimento e o trabalho desenvolvido no Brasil, denominado educação popular \& saúde.

Nossa participação, tanto na conferência quanto no curso somente foi possível pelo apoio recebido do Canadian Institutes of Health Research, para o pagamento integral de despesas (inscrição, hospedagem e transporte). Para tanto obtivemos ajuda da Prof. ${ }^{a}$ Margareht S. Zanchetta ${ }^{d}$, que, conhecedora de nosso interesse no tema educação popular \& saúde, nos incentivou e realizou esforços para que conhecêssemos 0 trabalho de educação de adultos naquele país e trocássemos experiências com pesquisadores da área.

No campo da saúde e direitos sexuais e reprodutivos sublinhamos a atividade junto a Colition. Trata-se de um grupo vinculado à Fédération du Québec pour le Planning des Naissances (FQPN); é constituído por representantes de grupos comunitários, de universidades e do movimento de mulheres. Tem como objetivo mobilizar a coletividade e denunciar a medicalização e a comercialização da saúde sexual e reprodutiva.

Tivemos a oportunidade de trocar experiências com representantes de movimentos organizados de mulheres e pesquisadoras(es) e profissionais. Os temas discutidos foram: cesariana eletiva; a banalização da anestesia peridural no processo de parto e nascimento; hormonioterapia; testes diagnósticos no período pré-natal para verificar anomalias cromossômicas, doenças hereditárias e malformação fetal, e, por último, a supressão da menstruação. Participamos ativamente desse evento, tanto no acompanhamento das discussões, quanto nas atividades dos trabalhos em grupo. Tivemos a oportunidade de apresentar alguns problemas considerados prioritários, em termos da saúde e dos direitos sexuais e reprodutivos das mulheres, no Brasil.

Ainda no âmbito da saúde e direitos sexuais e reprodutivos, ressaltamos a Conférence Anuelle 2004 de l'Association pour la Santé Publique du Québec - Obstétrique et Santé Publique. Este evento acontece anualmente como parte integrante das Journées Annuelles de Santé Publique (JASP), as quais objetivam a formação continuada e a troca de conhecimento entre os participantes (pesquisadores/as; professores/as; profissionais de diversas áreas; membro de associações e grupos comunitários, estudantes, entre outros). Trata-se do maior evento do gênero realizado no Quebec.

Destacamos como pontos relevantes da Conferência o alto nível das apresentações e as discussões relacionadas a alguns problemas, também comuns ao cenário brasileiro, como por exemplo: a cultura profissional que reforça o medo nas mulheres no processo do parto e nascimento, retirandoIhes a autonomia; a abordagem tecnológica excessiva e, muitas vezes, desnecessária e prejudicial, predominante nos serviços de saúde; e a necessidade de mudança no modelo assistencial no campo da atenção obstétrica. Foi possivel adquirir materiais informativos, boletins e livros, bem como realizar contatos com os conferencistas, membros de grupos comunitários e os outros participantes. 


\section{Atividades realizadas sob a forma de Visitas Técnicas a Instituições de Saúde e Organismos Comunitários}

Os locais visitados foram selecionados, tendo em vista os seguintes objetivos: a) conhecer a realidade do Quebec concernente à saúde em geral e à saúde e direitos sexuais e reprodutivos das mulheres; b) verificar a atuação de grupos de pesquisas, cuja problemática e objetos de estudos relacionavam-se à tese que desenvolvíamos; c) ampliar nosso conhecimento em termos de práticas em saúde voltadas para a humanização e respeito à autonomia das mulheres no campo da reprodução; e d) trocar experiências e conhecimento com profissionais e membros de grupos organizados na área da saúde da mulher, com atuação marcadamente comprometida com o fortalecimento da autonomia desta população.

Para realização desta atividade contamos com o auxílio do professor co-orientador que fazia contatos com as(os) "facilitadoras(os)", os quais num primeiro momento nos recebiam e, após delimitação dos nossos interesses, marcavam reuniões e/ou agendavam visitas com outras pessoas (contatos), grupos e serviços. Impressionaram-nos a receptividade, o empenho e a atitude carinhosa dessas pessoas no atendimento às nossas demandas. Chamou nossa atenção, também, o grande interesse desses(as) facilitadores(as) em relação ao Brasil, bem como seus conhecimentos sobre nossos problemas, em particular, no campo da saúde e, ainda, sobre as características culturais, sociais e nível de desenvolvimento político-econômico.

Este grupo de atividades enriqueceu substancialmente 0 universo de conhecimento acerca dos objetivos do estagio. 0 contato com as pessoas envolvidas e suas práticas e com os ambientes proporcionaram a incorporação de elementos fundamentais que nos permitiram, ainda que provisoriamente, compreender, comparar ou mesmo chegar a conclusões sobre os aspectos relacionadas à promoção da saúde e da qualidade de vida, mais especificamente das mulheres. Estes encontros possibilitaram, também, nossa aproximação com a problemática que envolve a atenção à saúde num contexto multicultural, como é o caso do Quebec.

\section{CONFERÊNCIAS: EM FOCO A SITUAÇÃO DA ATENÇÃO OBSTÉTRICA NO BRASIL}

Durante a realização deste estágio, tivemos a oportunidade de proferir duas conferências. Ambas sobre a temática: "Atenção Obstétrica no Brasil - avanços e perspectivas". A primeira conferência foi realizada na sede da ASPQ por ocasião da reunião do Comitê National d'Orinetation et de Mobilization en Perinatalité da própria ASPQ. Participaram representantes da ASPQ; da FQPN; dos CLSC; de Centres Hospitaliéres; grupos comunitários, pesquisadores(as) e profissionais, entre outros. Esta reunião foi de fundamental importância para a realização de contatos e trocas de informações com pessoas e grupos, organizados posteriormente.

A segunda conferência aconteceu durante a reunião científica do Département d'Obstétrique-gynécologie do Centre
Hospitalier de LaSalle, em que estiveram presentes professores, médicos e residentes do serviço. Após a apresentação discutiuse sobre a situação do Brasil em relação à atenção obstétrica, em particular, no que se refere à utilização de intervenções como 0 parto cirúrgico e sobre a morbimortalidade materna e perinatal. Nos dois momentos a facilitadora foi a Dra. Hélène Vadeboncoeur.

\section{CONTATOS RELEVANTES:}

Muitos contatos foram realizados durante o estágio. Uma grande quantidade de material educativo, tais como livros, panfletos informativos, periódicos canadenses e internacionais, foram adquiridos nestas oportunidades, bem como obtivemos a indicação de várias fontes de pesquisas.

Como contato individual, destacamos a Profa. Dr ${ }^{\mathrm{a}}$. Louise Dumas, professora e pesquisadora em Enfermagem na Université du Québec em Outaouais; primeira avaliadora do Quebec e do Canadá para a Iniciativa Hospital Amigo da Criança (IHAC). Reunimo-nos, durante quatro horas, em Longueuil (Quebec), para discutirmos sobre a situação da prática de aleitamento materno e da IHAC, no Brasil e no Quebec. Tivemos oportunidade de trocar muitas informaç̃̃es. Nesta oportunidade fizemos uma exposição sobre as ações voltadas para a proteção da prática da amamentação, no âmbito dos direitos sociais e trabalhistas. Trocamos informações sobre a política de incentivo ao aleitamento materno e sobre o processo de credenciamento dos hospitais na IHAC, tanto no Brasil quanto no Canadá.

Com relação aos grupos e/ou organismos comunitários, destacamos o Centre de Documentation sur l'Éducation des Adultes et la Condition Féminine (CDÉACF), a FQPN, que vincula grupos como a Coalition pour la Santé Sexuelle et Reproductive, todos anteriormente citados, e também o grupo Naissance et Renaissance, que, desde 1997, desenvolve ações com vistas à humanização do processo de parto e nascimento.

\section{AVALIAÇÃO DA ESTRUTURA BÁSICA, SISTEMAS E MÉTODOS DE TRABALHO E ATIVIDADES COMPLEMENTARES}

A UdeM é um dos mais importantes pólos de ensino superior e de pesquisa do Canadá, o primeiro do Quebec e um dos primeiros da América do Norte. A internacionalização de suas atividades, especialmente as ligadas à formação profissional, constitui-se numa de suas dimensões prioritária; coopera com mais de 50 parceiros no mundo e recebe cerca de 4.000 estudantes de outros países. No campo das ciências da saúde é a mais importante do Canadá.

A Faculté des Sciences Infirmières/UdeM, criada em 1962, tem lugar de destaque como líder em matéria de formação e pesquisa em Enfermagem nos países de língua francesa. 0 Programa de Doutorado, desenvolvido em conjunto com a Universidade McGill, tem como objetivo formar pesquisadores autônomos e aptos a contribuir para o desenvolvimento dos conhecimentos da Enfermagem como ciência. As pesquisas realizadas orientam-se pelo estudo do cuidado mediado pelas experiências de saúde do indivíduo, da família, de grupos e da comunidade com estreita interação destes com o ambiente em 
que se inserem. Favorece ainda a interdisciplinaridade e a colaboração nacional e internacional. Assim, a instituição pôde oferecer a infra-estrutura necessária para a realização do estágio.

Tivemos o regozijo de ter à nossa disposição um escritório individual na sede do GRASP, com equipamentos (computador, impressora, copiadora); recursos para comunicação (acesso a internet e telefone); recurso materiais (folhas para impressão, tinta para impressora, papel para rascunho e afins) e condições ambientais favoráveis (ambiente acolhedor, silencioso e amplo). A atitude democrática, comprometida e solidária do professor co-orientador estrangeiro, em relação às nossas dificuldades iniciais com a boa desenvoltura no idioma francês, permitiu que nos tranqüilizássemos e, paulatinamente, avançássemos no processo de aprendizagem e na segurança para nos expressar durante as aulas e publicamente. Chamou-nos à atenção o fato de que, em nossa relação, prevaleceram as nossas demandas e, por conta desta situação, mesmo nos momentos de muito cansaço físico ou mental, sentimo-nos motivadas a perseguir e alcançar os objetivos previamente traçados.

Em síntese, consideramos que a experiência foi bemsucedida e que conseguimos alcançar os objetivos propostos. A estrutura oferecida para pesquisa na UdeM foi excelente e a boa relação estabelecida com meu professor co-orientador estrangeiro foi fundamental para a realização desse estágio, especialmente do ponto de vista de minha inserção na vida acadêmica.

\section{CONCLUSÕES: DOS FATOS AO RELATO DA EXPERIÊNCIA}

Durante a realização do estágio tivemos a oportunidade de atualizar a revisão de literatura. Também foi possível verificar que as questões ligadas à conquista dos direitos sexuais e reprodutivos, em particular das mulheres, fazem parte de um movimento que extrapola as fronteiras brasileiras e, por assim ser, devem ser consideradas de uma maneira global, ao mesmo tempo em que solidária, entre os países e grupos interessados. Resguardadas as diferenças no plano de desenvolvimento político, cultural, econômico e social, apreendemos que as situações como: as iniqüidades de gênero, o acesso prejudicado a bens sociais e a cultura discriminatória, que submetem as mulheres a dificuldades de toda ordem na obtenção de qualidade de vida e saúde, extrapolam as fronteiras dos países, atingindo de forma diferenciada - mas nem por isso menos grave - mulheres e homens em várias partes do planeta.

As discussões, leituras e reflexões realizadas proporcionaram ampliação significativa na compreensão de um dos conceitos mais importantes de nossa tese, qual seja, a assunção mesma de que a saúde sexual e reprodutiva deve ser entendida na perspectiva do necessário desenvolvimento e garantia dos direitos humanos. Isto se deve ao fato tácito de que este campo da saúde tem íntima relação com as iniqüidades, às injustiças sociais e, fundamentalmente, com a forma com que as sociedades, influenciadas por questões culturais e econômicas, cuidam ou não das mulheres e das questões associadas à sua saúde e vida reprodutiva.
Participamos de quase todos os eventos disponíveis em nosso campo de interesse. 0 professor co-orientador estrangeiro acompanhou todas as atividades, incentivando-nos, orientandonos e auxiliando-nos na resolução de problemas simples do cotidiano - abertura de conta bancária; procura de locais para moradia nas duas oportunidades em que foi necessário; adaptação à universidade e à cidade e, finalmente, na minha integração social e inserção na vida acadêmica. Consideramos que esta importante ajuda foi fundamental para o bom desenvolvimento do estágio, pois nos proporcionou sentimentos de segurança e tranqüilidade. A receptividade e a cordialidade das pessoas, de uma forma geral, também foram fatores de grande importância para o bom desenvolvimento do trabalho.

$A$ adaptação tranqüila à vida acadêmica e ao cotidiano da cidade de Montreal foi um fator positivo para o desenvolvimento de todas as atividades realizadas. 0 receio quanto à habilidade de comunicação no idioma francês foi superado com a realização de curso do idioma francês; com a participação em sala de aula e em eventos e reuniões e, finalmente, nas relações interpessoais. Durante esta experiência contamos, ainda, com o apoio dos(as) amigos(as) brasileiros(as), das colegas de curso, como também dos(as) novos(as) amigos(as) que conhecemos em Montréal.

Cabe-nos ainda ressaltar aos(as) estudantes de doutorado deste país que a realização de intercâmbio com instituições internacionais de ensino e pesquisa vem ao encontro dos desafios que urgem ser superados, tanto do ponto de vista do avanço do conhecimento, quanto da formação de novos(as) pesquisadores(as), visando ao progresso científico, tecnológico, econômico e social de nosso país. Nesta perspectiva, vale referir a importância da formação de recursos humanos com competência para superação dos desafios em um país em desenvolvimento, como é o caso do Brasil.

Acreditamos que esta experiência poderá motivar outros(as) estudantes do Programa de Pós-graduação de minha escola de origem e, assim, contribuir para a melhoria da qualidade do mesmo. Ressaltamos que esta experiência fortaleceu, substancialmente, nosso intuito de inserirmo-nos no "mundo" da pesquisa como uma prática de desenvolvimento profissional e pessoal. Finalmente, reiteramos que atravessar as fronteiras do Brasil para melhor apreender os desafios que se impõem para as mudanças urgentes, em especial no campo da saúde, foi uma experiência relevante e, cremos, embrionária de outras experiências. Assim, nos demos conta ainda de um dos grandes desafios da Enfermagem brasileira e mundial contemporânea - (re)contruir novas práticas no campo da assistência, do ensino e da pesquisa, conjugando arte e ciência e transformando a própria enfermagem na ação de se (re)fazer, (re)pensar e (re)produzir no âmbito da saúde e da vida.

Aproveitamos para registrar nossos agradecimentos a todas as instituições, em particular a CAPES/MEC, e às pessoas citadas, em especial à Dra. Hélène Vadeboncouer por sua disponibilidade em nos auxiliar e acompanhar nesta trajetória, bem como a outras instituições e pessoas que, embora não tenham sido referidas, integram o rol dos que merecem o nosso mais profundo reconhecimento. 


\section{Referências}

1 Ministério da Educação (BR). Coordenação de Aperfeiçoamento de Pessoal de Nivel Superior-CAPES [site da Internet]. Estágio de Doutorando-Balcão (sanduíche/sandwich) [acesso 18 fev 2006]. Disponível em: http//www.capes.gov.br/bolsas/noexterior/ estagio_doutorandobalcao.html.

2 Souza KV. A saúde da mulher e seus direitos reprodutivos em um "círculo de cidadania" [tese de doutorado]. Rio de Janeiro (RJ): Escola de Enfermagem Anna Nery/UFRJ; 2005.

3 Carvalho V. Dia 20 de maio de 2004 - Jubileu de ouro da classe de 1954: discurso de saudação e relatos de experiências. Esc Anna Nery Rev Enferm 2004 ago; 8 (2): 291-97.

\section{Notas Bibliográficas}

a Professor Titular da EEAN/UFRJ. Membro do NUPESM/DEMI/EEAN/ UFRJ. Diretora da EEAN/UFRJ.

b PhD em Sociologia. Professor Titular da Faculdade de Enfermagem, da Universidade de Montreal (Quebec/Canadá).

c Relatório de Atividades de Doutorado Sanduíche aprovado pela CAPES. d Professor Adjunto da Escola de Enfermagem/Univerdade de Ryerson (Toronto/Canadá).

e Pesquisadora, PhD em sociologia. Membro do Centro de Pesquisa e de Formação do CLSC Côte-des-Neiges, 2001-2005.

f Hélène Vadeboncoeur, Ph.D. em Ciências humanas, pela UdeM . É agente de pesquisa em perinatologia desde a década de 90 . Autora do livro "Une autre césarienne? non, merci!" (1989); colaborou com a obra Re-conceiving Midwifery (McGill-Queen's University Press, 2004) e artigos publicados em periódicos científicos internacionais. 